\title{
Estimation of thermophysical properties of thin plates with averaging techniques and two temperatures model
}

\author{
by J.C. Batsale, D. Mourand, C. Gobbé
}

Laboratoire Energétique et Phénomènes de Transfert- ENSAM (URA CNRS 873), Esplanade des Arts et Métiers, 33405 Talence Cedex - France

Tél: (33)56 845425 - Fax: (33)56 845401

Abstract

Averaging techniques are well suited for infrared image processing because they consider spatially averaged temperature fields related or not to each constitutive phase of heterogenous media. In the case of simple geometry, such as thin rectangular adjacent plates, one or two dimensional exact analytical relationships can be obtained between averaged temperature related to each plate. These expressions are suitable to estimate thermophysical properties such as thermal diffusivity or thermal resistance at the junction of the plates. The main advantage is to reduce the influence of measurement noise. Experimental results are obtained and some examples of estimation of thermophysical parameters are presented.

\section{Introduction}

Two-temperatures models are generally used to describe diffusive transfer in porous media at macroscopic scale in the case of non-local thermal equilibrium (see Vortmayer and Schaefer [1], Schlunder [2]). Recently, Quintard and Whitaker [3] have revisited these models and better justified several assumptions, especially the exchange coefficient between local phases, with averaging techniques.

Two-temperature models are not commonly used to interpret experimental data because of the difficulty in estimating the average temperatures. However, infrared image processing is well suited for such an interpretation task since enough data are available to compute these averaged fields.

In the case of simple geometry, such as the thin rectangular layered plates shown in Figure 1, one or two dimensional exact analytical relationships between the phase averaged temperatures can be obtained in order to estimate thermophysical properties such as thermal diffusivity or interfacial resistances.

2 Analytical relationships between averaged quantities in the case of two adjacent rectangular plates

Consider two adjacent rectangular thin plates whose geometry is presented in figure 1. The experiment consists in heating the plate 1 (for example by transient Joule effect). The thermal excitation must be uniform in the $x$-direction and only a function of $y$ and $t$.

The temperature gradient is assumed to be uniform perpendicular to $x-y$ plane. Lateral heat losses are considered to be uniform in the $x-y$ plane. This results in a source term proportional to the temperature field (fin hypothesis).

The general system governing heat transfer in both adjacent plates can then be written:

$$
\begin{aligned}
& \frac{\partial^{2} T_{i}}{\partial x^{2}}+\frac{\partial^{2} T_{i}}{\partial y^{2}}-\frac{2 h}{\lambda_{i} e}+\frac{Q_{i}(y, t)}{\lambda_{i}}=\frac{1}{a_{i}} \frac{\partial T_{i}}{\partial t} \quad I=1,2 \\
& \frac{\partial T_{1}}{\partial x}=0 \quad \text { at } x=-\mathrm{e} \\
& -\lambda_{1} \frac{\partial T_{1}}{\partial x}=-\lambda_{2} \frac{\partial T_{2}}{\partial x} \quad \text { and } T_{1}=T_{2} \quad \text { at } x=0
\end{aligned}
$$




\section{http://dx.doi.org/10.21611/qirt.1996.008}

$\frac{\partial T_{2}}{\partial x}=0$

at $x=\mathrm{e}_{2}$

Boundary conditions at $y=0$ and $y=L$ are assumed to be homogenous and will be explained for each particular case. Integral Laplace transform on variable $t$ and Fourier transform on $y$ (Sine, Cosine or exponential Fourier transform following boundary conditions imposed at $y$ ends), give the transformed general equation (1-a) such as:

$$
\begin{aligned}
& T_{i}=0 \\
& \quad-\beta_{i}^{2} \theta_{i}+\frac{d^{2} \theta_{i}}{d x^{2}}+\frac{F}{\lambda_{i}}=0 \quad \text { With: } \beta_{i}^{2}=\left(\frac{p}{a_{i}}+\alpha^{2}+\frac{2 h}{\lambda_{j} e}\right)
\end{aligned}
$$

where $\alpha$ is the spatial frequency in the transformed space following $y, p$ is the Laplace variable and $F(s, p)$ or $F(\alpha, p)$ corresponds to successive transformation of $Q(y, t)$. Solution of this problem can be presented, for phase 1, under such quadrupolar form (See [6] for further informations about quadrupole method and integral transforms):

$\left(\begin{array}{l}\theta_{1}(x, p, \alpha) \\ \phi_{1}(x, p, \alpha)\end{array}\right)=\left(\begin{array}{cc}\operatorname{ch}\left(\beta_{1} x\right) & -\frac{\operatorname{sh}\left(\beta_{1} x\right)}{\lambda_{1} \beta_{1}} \\ -\lambda_{1} \beta_{1} \operatorname{sh}\left(\beta_{1} x\right) & \operatorname{ch}\left(\beta_{1} x\right)\end{array}\right)\left(\begin{array}{c}\theta_{1}(0, p, \alpha)+F(\alpha, p) \frac{\left(\operatorname{ch}\left(\beta_{1} x\right)-1\right)}{\lambda_{1} \beta_{1}^{2}} \\ \phi_{1}(0, p, \alpha)+F(\alpha, p) \frac{\operatorname{sh}\left(\beta_{1} x\right)}{\beta_{1}}\end{array}\right)$

Averaged temperature and flux along $x$ are defined as:

$<\theta_{1}>^{1}=\frac{1}{e_{1}} \int_{0}^{e_{1}} \theta_{1}(x, p, \alpha) d x ;<\phi_{1}>^{1}=\frac{1}{e_{1}} \int_{0}^{e_{1}} \phi_{1}(x, p, \alpha) d x$

vector $\left.\left.\left(<\theta_{1}\right\rangle^{1},<\phi_{1}\right\rangle^{1}\right)^{t}$ can be related to the characteristic vector of the interface between the two plates $\left(\theta_{1}\left(e_{1}, \alpha, p\right) ; \phi_{1}\left(e_{1}, \alpha, p\right)\right)^{t}$, with the following quadrupolar expression:

$\left(\begin{array}{l}<\theta_{1}>^{1} \\ <\phi_{1}>^{1}\end{array}\right)=\left(\begin{array}{cc}1 & \frac{1}{\lambda_{1} \beta_{1} t h\left(\beta_{1} e_{1}\right)}-\frac{1}{\lambda_{1} \beta_{1}^{2} e_{1}} \\ \lambda_{1} \beta_{1}^{2} e_{1} & \frac{\beta_{1} e_{1}}{t h\left(\beta_{1} e_{1}\right)}\end{array}\right)\left(\begin{array}{l}\theta_{1}(0, \alpha, p) \\ \phi_{1}(0, \alpha, p)\end{array}\right)$

Similar procedure for plate 2 gives:

$\left(\begin{array}{l}\theta_{2}(0, \alpha, p) \\ \phi_{2}(0, \alpha, p)\end{array}\right)=\left(\begin{array}{cc}\frac{\beta_{2} e_{2}}{\operatorname{th}\left(\beta_{2} e_{2}\right)} & \frac{1}{\lambda_{2} \beta_{2}{ }^{2} e_{2}}-\frac{1}{\lambda_{2} \beta_{2} t h\left(\beta_{2} e_{2}\right)} \\ -\lambda_{2} \beta_{2}{ }^{2} e_{2} & 1\end{array}\right)\left(\begin{array}{l}<\theta_{2}>^{2} \\ <\phi_{2}>^{2}\end{array}\right)$

If contact between plates is uniform (non-depending on $y$ ), interface transfer yields:

$\left(\begin{array}{l}\theta_{1}(0, \alpha, p) \\ \phi_{1}(0, \alpha, p)\end{array}\right)=\left(\begin{array}{cc}1 & R_{c} \\ 0 & 1\end{array}\right)\left(\begin{array}{l}\theta_{2}(0, \alpha, p) \\ \phi_{2}(0, \alpha, p)\end{array}\right)$

Expressions (5) and (6) can be illustrated by an analogic electrical scheme (figure 2) which shows that the exchanged flux between phase 1 and 2 can be written as:

$\left.\left.<\phi>=Z\left(<\theta_{1}\right\rangle^{1}-<\theta_{2}\right\rangle^{2}\right)$

with $Z$, impedance defined by:

$Z=\frac{1}{\lambda_{1} \beta_{1} t h\left(\beta_{1} e_{1}\right)}-\frac{1}{\lambda_{1} \beta_{1}^{2} e_{1}}+R_{c}+\frac{1}{\lambda_{2} \beta_{2} t h\left(\beta_{2} e_{2}\right)}-\frac{1}{\lambda_{2} \beta_{2}{ }^{2} e_{2}}$

Expression (8) models the global thermal exchange between phase 1 and phase 2 . The first order Taylor expansion of $Z$ ( with $\beta$ small) gives a pure resistance : 
$Z \approx \frac{e_{1}}{3 \lambda_{1}}+\frac{e_{2}}{3 \lambda_{2}}+R_{c}$

If the rectangular plates are considered as one elementary cell of a periodic porous medium, the approximation of $Z$ can be used to model heat transfer in porous media $([1,2])$ and has been rigorously defined in [3]. $Z$ describes the thermal exchange between phase 2 and phase 1 . Such a rectangular geometry is a suitable situation to test parameters estimation. Several direct applications in this case can be implemented in the case of non-destructive testing of welding between thin metallic plates.

\section{Identification method in the case of 1D system}

\subsection{Explicit identification formula}

In the experimental case presented on figure 1 , the heat transfer can be considered as one dimensional. The heat excitation is uniform in the $y$ direction and adiabatic conditions are assumed at the $y$-ends. Then, the spatial frequency $\alpha$ is zero and temperature field is averaged not only on $x$, but also on $y$.

In zone 2, no heat excitation occurs, and the averaged heat flux in zone 2 can be expressed as: $\left\langle\phi_{2}\right\rangle^{2}=0$

This last expression replaced in (6) gives one simple relationship between averaged temperatures in each parts:

$$
<\theta_{1}>^{1}=\left(1+Z \lambda_{2} \beta_{2}{ }^{2} e_{2}\right)<\theta_{2}>^{2} \quad \text { with } \beta_{i}{ }^{2}=\frac{p}{a_{i}}+\frac{2 h}{\lambda_{j} e}
$$

Expression (12) is convenient because the knowledge of $Q(t)$ is here not necessary to estimate parameters such as $a_{1}$. or $R_{G}$. Moreover, emissivity does not need to be known, if this parameter is uniform on both plates. Only a signal proportional to the temperature field has to be processed.

For example, in the simple case where plate 1 is the same as plate 2 with perfect contact, thermal diffusivity can be estimated. This will be shown on the further example.

\subsection{Estimation with two temperatures model}

If plate 1 is the same as plate 2 and with perfect contact, combination of expression (12) and $(10)$ gives:

$$
<\theta_{1}>^{1} \approx(A p+B)<\theta_{2}>^{2} \quad \text { With } A=\frac{2 e_{1}^{2}}{3 a_{1}} \text { and } B=1+\frac{4 h e_{1}}{3 \lambda_{1} e}
$$

Coefficients $A$ and $B$, can be identified by processing $\left.\left(\left\langle\theta_{1}\right\rangle^{1} /<\theta_{2}\right\rangle^{2}\right)$ for several $p$ values. This last function is then a straight line of slope $A$ and origin $B$. The first parameter $A$ is only depending on thermal diffusivity and the second parameter $B$ depending on the heat losses non-correlated with $A$. $p$ values are chosen in a convenient interval in order to avoid zero dividing at short time and truncation error of the Laplace integral at long time (see [7]).

\subsection{Use of minimisation algorithm with exact model}

After the first estimation of thermal diffusivity and heat losses by direct expression (13), it is possible to improve the estimation by a minimisation algorithm.

Expression (13) can be written in time space with a convolution product. The inverse transform of the transfer function between $\left\langle\theta_{1}\right\rangle^{1}$ and $\left\langle\theta_{2}\right\rangle^{2}$ is in time space:

$$
G(t)=L^{-1}\left(\frac{1}{1+Z \lambda_{2} \beta_{2}{ }^{2} e_{2}}\right)=L^{-1}\left(\frac{\operatorname{th}\left(\beta_{1} e_{1}\right)}{2 \beta_{1} e_{1}-t h\left(\beta_{1} e_{1}\right)}\right)
$$

Expression (4) can then be written in time space: 


\section{http://dx.doi.org/10.21611/qirt.1996.008}

$<T_{2}>^{2}(t)=\int_{0}^{t}<T_{1}>^{1}(t-\mu) G(\mu) d \mu$

The first estimation of parameters by the previous explicit formulas can give the first step of a minimisation algorithm (Nelder-Mead or function fmins in Matlab [5]). The quantity to be minimised is the difference between the theoretical evaluation of $\left\langle T_{2}\right\rangle^{2}(t)$ by the convolution product (16) and the experimental values of $\left\langle T_{2}\right\rangle^{2}(t)$.

\section{Rough estimation in 2D case}

If the contact between the plates is non-uniform (depending on $y$ ), the interface transfer expression is more complicated in transformed space (see [4]). Therefore, expression (5) and (6) can even be used, in order to process averaged quantities instead of local noisy data.

At short time the Laplace variable can be considered as: $\frac{p}{a_{i}}>\frac{2 h}{\lambda_{j} e}$ and $\frac{p}{a_{i}}>\alpha^{2}$, this is equivalent to assume that lateral heat losses and heat diffusion in the $y$-direction are negligible. In the case where plate 1 is the same as plate 2 with $e_{3}=e_{2}$, the following expressions can be written from (5)and (6):

$$
R_{c}(y)=\frac{\left(<T_{1}>^{1}(t, y)-<T_{2}>^{2}(t, y)\right)}{\rho c_{2} e_{2}\left(\frac{\partial<T_{2}>^{2}}{\partial t}(t, y)\right)} \text { or } R_{c}(y)=\frac{\int_{0}^{t_{i}}\left(<T_{1}>^{i}(t, y)-<T_{2}>^{2}(t, y)\right) d t}{\rho c_{2} e_{2}\left(<T_{2}>^{2}\left(t_{i}, y\right)-<T_{2}>^{2}(0, y)\right)}
$$

As previously, such expressions are independent on time evolution of the heat excitation and do not need the knowledge of emissivity.

\section{Experiment}

\subsection{General device}

Experimental results have been obtained with infrared camera TVS 2000 from AVIO. The maximal scanning rate is 30 images/s and each frame is $100 \times 256$ pixels. Each experiment is composed of more than 100 frames recorded at regular time steps. The entire sequence is stored after the experiment from the real-time memory of the thermographic device into a magneto-optic disk. Then the experimental data are directly loaded and processed in Matlab [5] on a PC with Pentium 100 processor. Some examples of images obtained after the beginning of heating are shown in figure 3. The processed surface of the sample is painted uniformly in black in order to avoid any emissivity variation. The transient form of heating is a slot of several second length. The energy is about $5 \mathrm{~W}$ on the surface of plate 1 . Then the maximum temperature difference is about $10 \mathrm{~K}$.

\subsection{Thermal diffusivity estimation-Plates with perfect contact(1D case)}

The sample to be tested is an aluminium alloy plate of thickness $e=2 \mathrm{~mm}$ and lateral size $e_{1}=e_{2}=32 \mathrm{~mm}$. The evolution of experimental averaged temperatures is presented in figure 4 . It can be seen that the noise influence on these curves is reduced compared to images (even smoothed). Processing of curves from figure 4 allows to first estimate parameters $A$ and $B$. Then, the thermal diffusivity $a$ is obtained with a minimisation algorithm. It can be seen on figure 4 that the theoretical evaluation of $\left\langle T_{2}\right\rangle^{2}(t)$, from experimental evolution of $\left\langle T_{1}\right\rangle^{1}(t)$ and convolution product (16), is well superposed to experimental values of $\left\langle T_{2}\right\rangle^{2}(t)$. We obtain here $a=4.710^{-5} \mathrm{~m}^{2} \mathrm{~s}^{-1}$ which has been confirmed by the classical flash method . 


\title{
5.3 2D case non uniform interface between plate 1 and 2
}

The sample to be tested is the same plate as in the previous case, but there is a non uniform split at the interface between the two plates (see figure 5). One example of image obtained during the excitation is given in figure 6. Application of the simple explicit expression (16) gives a first rough estimation of the $y$-distribution of the interface resistance. The profile is fitting to the slit geometry, even if too restrictive approximations do not allow to obtain the right local values.

\section{Conclusion}

Advantages of such methods are that even poor experimental information is sufficient to process the estimation (emissivity, evolution of the heat excitation). Averaging is here efficient to reduce the influence of measurement noise. Perspectives are to improve estimation in $2 \mathrm{D}$ cases, by taking into account lateral heat losses and 2D diffusion.

\section{Nomenclature:}

$a_{i}$ : thermal diffusivity of phase $i$

$e_{i}$ : width of plate $i$

e.thickness of the plate

$\beta_{i}$ :generalised frequency relative to phase $i$

G:transfer function

$h$ :lateral convective exchange coefficient

$L^{-1}$ : Inverse Laplace transform

$\lambda_{j}$ : thermal conductivity of phase $i$

\author{
p: Laplace variable \\ $Q, F:$ Heat excitation, (real, transformed) \\ $(\rho c)$ i: volumic heat of phase i \\ $t$ time \\ $\left\langle T_{i}:\right\rangle^{i},\left\langle\theta_{i}\right\rangle^{i}$ averaged temperature (real, transf.) \\ $Z$ : exchange impedance
}

\section{REFERENCES}

[1] Vortmeyer D. and Schaeffer R.J - Equivalence of one and two phase models for heat transfer processes beds: one dimensional theory, Chem. Eng. Sci. 29 (1974) pp. 485-491.

[2] Schlunder E - Equivalence of one and two phase models for heat transfer processes in packed dimensional theory, Chem. Eng. Sci. 30, (1975) pp 449-452.

[3] Quintard M., Whitaker S.- Transport in ordered and disordered porous media part I,II,II,,IV,V. Transport in Porous Media, 14, pp 163-206, 15, (1994) pp31-71 and pp 183-196.

[4] Batsale J.C., Maillet D., Degiovanni A. Extension dela méthode des quadripoles thermiques à l'aide de transformation integrales-Application au defaut plan bidimensionnel-Int.Journal of Heat and Mass Transfer, Val 37, n1, (1994) pp111-127

[5] Matlab User's guide: The Mathworks, Inc.-21 Eliot Street- South Natick, MA 01760

[6] J.C. Batsale, B. Ladevie, J. Gounot: Measurement of macroscopic thermophysical properties of geological core samples by infrared image processing.-2nd Int. Conf. on Inverse Problems in Engineering; theory and practice-June 96-Le Croisic -France

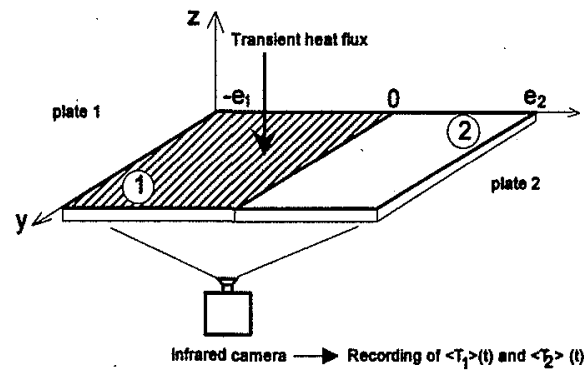

Fig 1: Experimental arrangement

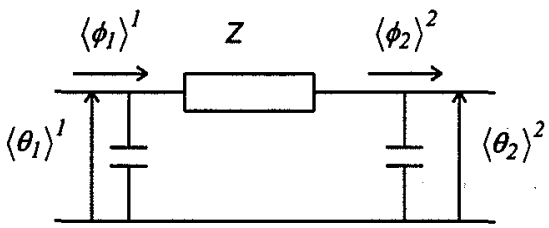

Fig 2: Analogy between average temperatures 

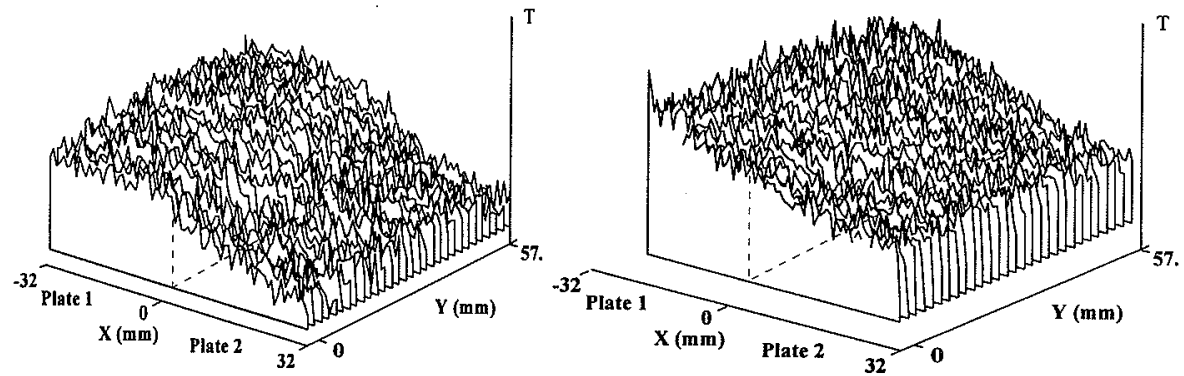

Fig 3: Images from $1 \mathrm{D}$ transient case. at $\mathrm{t}=5 \mathrm{~s}$ and $\mathrm{t}=16.6 \mathrm{~s}$.

(smoothed with a moving average of $3^{\star} 3$ pixels)

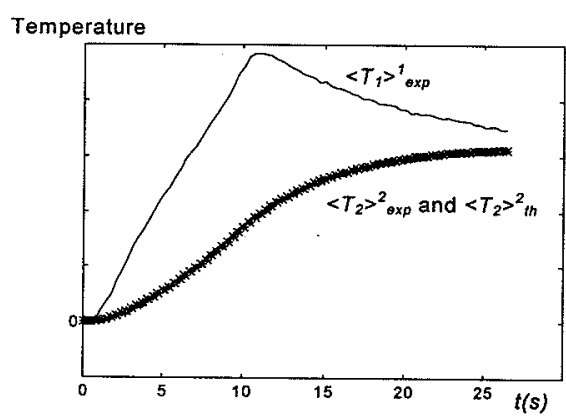

Fig 4 Averaged temperature evolutions in 1D case

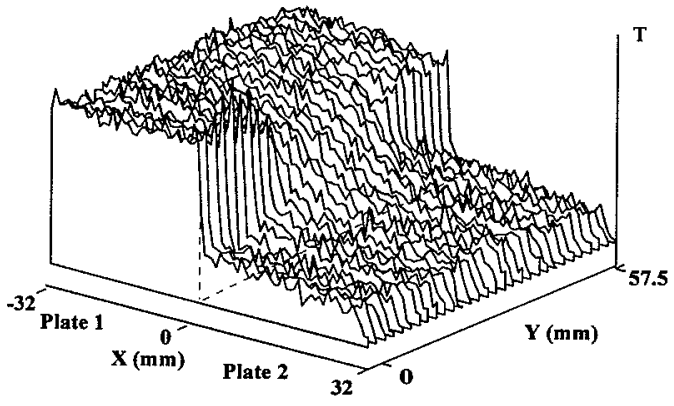

Fig 6: Image obtained in 2D transient case $(t=15 s)$ (smoothed with a moving average of $3^{*} 3$ pixels)

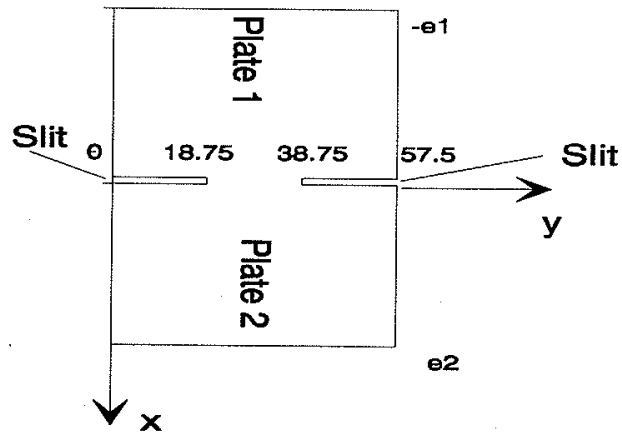

Fig 5: Non uniform interface

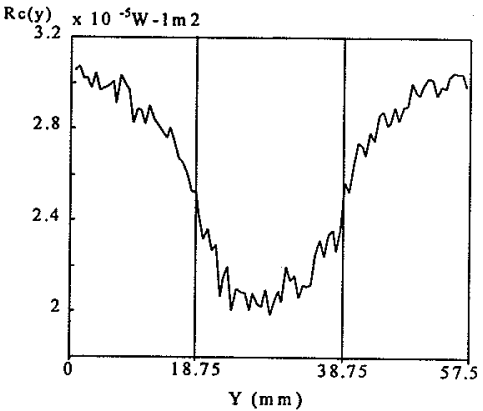

Fig 7: Estimation of interface profile ( $\mathrm{ti}=13 \mathrm{~s})$ 